\title{
Treatment Outcome of Gamma Knife Radiosurgery of Vestibular Schwannomas with Cystic Component
}

\author{
Jung Min Lee, Do Hoon Kwon, Chang Jin Kim, Jeong Hoon Kim \\ Department of Neurosurgery, Asan Medical Center, University of Ulsan College of Medicine, Seoul, Korea
}

\begin{abstract}
Objective: Many studies have shown favorable outcome of Gamma knife radiosurgery (GKRS) for vestibular schwannomas (VSs). However, there have been few studies which have shown the treatment result of cystic VSs (CVSs) for GKRS. The aim of this study was to confirm whether GKRS for CVSs continues to be safe and effective.

Methods: The study population consisted 511 patients (172 males, 339 females) with VSs treated with GKRS between May 1990 and December 2013. Group 1 defined as cyst/tumor volume $>30 \%$, group 2 was cyst/tumor volume $>50 \%$, and group 3 was solid tumor or cyst/tumor volume $\leq 30 \%$. To analyze factors that correlated with treatment outcomes, the following factors were assessed: tumor volume, cyst volume, tumor/cyst ratio, cyst type (single vs. multicystic), previous resection history, marginal radiation dose.

Results: The median follow up period was 52 months (range, 12-110 months). Tumor progression in group 1 was $5(18.5 \%)$, group 2 was $1(11.1 \%)$ and group 3 was $31(6.4 \%)$. In group 1, 2 patients underwent delayed microsurgery at 31 and 36 months after GKRS.

Conclusion: In this study we found that GKRS for large CVS was associated with poor treatment outcome. Further study is needed to identify the mechanism of the tumor progression of CVS after GKRS
\end{abstract}

Key Words: Gamma knife $\cdot$ Cystic vestibular schwannoma $\cdot$ Solid vestibular schwannoma

\section{INTRODUCTION}

Vestibular schwannomas (VSs) are relatively common intracranial tumors, account for $10 \%$ of all primary intracranial tumors $^{13)}$, are the most common tumors occupying the cerebellopontine angle, and accounts for $80 \%$ of cerebellopontine angle tumors. Numerous studies have reported incidence rates ranging from 0.2 to 1.7 per 100,000 population ${ }^{7}$.

Based on their consistency, they can be broadly grouped into cystic VSs (CVSs) and solid VSs (SVSs) ${ }^{12)}$. CVSs are believed by some to have a more aggressive course with more rapid neurological deterioration and have less favorable surgical outcomes than SVSs with regard to facial nerve outcomes, surgery-related complications, and mortality ${ }^{1,3)}$.

It is widely believed that CVSs have less favorable surgical outcomes than SVSs with regard to facial nerve outcomes, surgery-related complications ${ }^{12}$. And the Marseille group reported treatment failure rate of CVS treated with Gamma knife radio-

Corresponding author: Do Hoon Kwon, MD, PhD

Department of Neurosurgery, Asan Medical Center, University of Ulsan College of Medicine, 13, Gangdong-daero, Songpa-gu, Seoul 05505, Korea Tel: +82-2-3010-3550, Fax: +82-2-476-6738

E-mail: dhkwonasan@gmail.com surgery (GKRS), was 6.4\%, 3 times what they report for SVSs ${ }^{5)}$. There are few studies which showed factors affecting the treatment result of CVSs for GKRS. The aim of this study was to confirm which factors affect the tumor progression after GKRS of CVSs.

\section{MATERIALS AND METHODS}

A consecutive series of 620 patients with VSs underwent GKRS between May 1990 and December 2013. Patients follow up less than 12 months by magnetic resonance image (MRI) were excluded. We used the GKRS to treat 511 patents affected by VSs, with median follow-up period 52 (range, 12110) months. Group 1 defined as cyst/tumor volume $>30 \%$, group 2 was cyst/tumor volume $>50 \%$, and group 3 was solid tumor or cyst/tumor volume $\leq 30 \%$.

All radiosurgery procedures in this study were carried out using a Leksell Gamma knife (B. and C. Elekta, Stockholm, Sweden). After a Leksell stereotactic coordinate frame was applied to the patient's head, MR T1-weighted images with gadolinium contrast were obtained, and slices were reconstructed every $2 \mathrm{~mm}$ in the axial plane. GammaPlanElecta (Elekta) Instruments software was used to plan GKRS. The median tumor marginal dose was $12 \mathrm{~Gy}$ (range, 11-13.5 Gy) with a median 
isodose lines of 50\% in all cases.

MRI was performed to evaluate the radiological response to GKRS every 6 to 12 months. We considered treatment outcome as regression, stable and progression, referred by generally accepted criterion ${ }^{9}$. All patients underwent preoperative magnetic resonance

\section{RESULTS}

Group 1 included 8 male (29.6\%) and 19 female (70.4\%), with a median age of 59 at time of treatment (range, 38-79 years). Two patients had undergone previous incomplete resection, and one patient had prior ventriculoperitoneal shunt (VPS) placement. Group 2, selected from group 1, includes 3 male (33.3\%) and 6 female (66.7\%), with a median age of 60 at time of treatment (range, 44-79 years). One patient had undergone incomplete resection and one patient had prior VPS placement. Group 3 includes 164 male (33.9\%) and 320 female (66.1\%), with median age of 53 at time of treatment (range, 24-78 years). Fifty-one patients had undergone incomplete resection (Table 1).

The efficacy of radiosurgery was assessed by observation of tumor's maximal diameter changes in all 511 VS patients who underwent regular MRI follow-ups (Tables 2, 3). During the follow-up, 20 patients (74.1\%) had a regression, 2 patients (7.4\%) had a stable response, and 5 patients (18.5\%) had a

Table 1. Baseline characteristics of the patient with vestibular schwannomas

\begin{tabular}{llrrr}
\hline \hline Variable & & Group 1 & Group 2 & \multicolumn{1}{c}{ Group 3 } \\
& & $27(100 \%)$ & $9(100 \%)$ & $484(100 \%)$ \\
\hline \multirow{2}{*}{ Sex' } & $M$ & $8(29.6 \%)$ & $3(33.3 \%)$ & $164(33.9 \%)$ \\
\multirow{2}{*}{ Age } & $F$ & $19(70.4 \%)$ & $6(66.7 \%)$ & $320(66.1 \%)$ \\
& Range & $38-79$ years & $44-79$ years & $24-78$ years \\
Side & Median & 59 years & 60 years & 53 years \\
& Right & $14(51.9 \%)$ & $5(55.6 \%)$ & $256(52.9 \%)$ \\
Previous & Left & $13(48.1 \%)$ & $4(44.4 \%)$ & $228(47.1 \%)$ \\
operation & Yes & $3(11.1 \%)$ & $1(11.1 \%)$ & $51(10.5 \%)$ \\
\hline
\end{tabular}

progression in group 1. In group 2, 6 patients (66.7\%) had a regression, 2 patients (22.2\%) had a stable response, and 1 patient (11.1\%) had a progression. And in group 3, 266 patients (54.9\%) had a regression, 187 patients (38.6\%) had a stable response, and 31 (6.4\%) had a progression. In group3, when follow-up period was more than two years, progression was 24 (5\%). Among increased tumor, two patients underwent surgical removal of the tumor in group1, one and three in group 2, 3 respectively, due to combined neurological deterioration. The median interval time from GKRS to tumor resection was 51 months (32-72 months).

Table 4 shows progression tumors after GKRS in group 1. These five patients were female and right side dominant with median age 66.8 (range, 59-79 years) and one patient was also belong to group 2 . Two underwent delayed microsurgery at 31 and 37 months after GKRS and the size of the tumor were 5.5 and $14.3 \mathrm{~cm}^{3}$, respectively. The histology study of the tumors showed typical benign VS in these two patients. Consider the median tumor volume in group 1, 2 and 3, we could conclude the reason for higher progression rate in group 1,2

Table 2. Radiologic features of vestibular schwannomas

\begin{tabular}{lccc}
\hline \hline Variable & Group 1 & Group 2 & Group 3 \\
\hline $\begin{array}{l}\text { Median tumor } \\
\text { volume }\left(\mathrm{cm}^{3}\right)\end{array}$ & 5.9 & 7.5 & 2.1 \\
$\begin{array}{l}\text { Median tumor cyst } \\
\text { volume }\left(\mathrm{cm}^{3}\right)\end{array}$ & 2.7 & 6.1 & \\
$\begin{array}{l}\text { Median tumor/cyst } \\
\text { volume ratio (\%) }\end{array}$ & $44(31-93)$ & $77(50-93)$ & \\
$\begin{array}{l}\text { Median tumor } \\
\text { marginal dose (Gy) }\end{array}$ & 12 & 12 & 12 \\
\hline
\end{tabular}

Table 3. Treatment outcome of Gamma knife radiosurgery for vestibular schwannomas

\begin{tabular}{lccc}
\hline \hline Treatment outcome & Group 1 & Group 2 & Group 3 \\
\hline Regression & $20(74.1 \%)$ & $6(66.7 \%)$ & $266(54.9 \%)$ \\
Stable & $2(7.4 \%)$ & $2(22.2 \%)$ & $187(38.6 \%)$ \\
Progression & $5(18.5 \%)$ & $1(11.1 \%)$ & $31(6.4 \%)$ \\
2nd treatment & $2(7.4 \%)$ & $1(11.1 \%)$ & $3(0.6 \%)$ \\
\hline
\end{tabular}

Table 4. Tumor progression after Gamma knife radiosurgery of vestibular schwannoma with cystic component

\begin{tabular}{|c|c|c|c|c|c|c|c|c|c|c|}
\hline Case & Sex & Age & Site & $\begin{array}{c}\text { Tumor } \\
\text { volume }\left(\mathrm{cm}^{3}\right) \\
\end{array}$ & $\begin{array}{c}\text { Cyst } \\
\text { volume }\left(\mathrm{cm}^{3}\right) \\
\end{array}$ & $\begin{array}{l}\text { Cyst/Tumor } \\
\text { volumeratio }\end{array}$ & $\begin{array}{c}\text { Prior } \\
\text { operation } \\
\end{array}$ & $\begin{array}{c}\text { GKRS protocol } \\
\text { (Gy) }\end{array}$ & $M x$ & $\begin{array}{l}\text { Interval } \\
\text { (months) }\end{array}$ \\
\hline 1 & $M$ & 59 & Left & 5.5 & 1.8 & $33 \%$ & $O$ (VPS) & 12 & Craniotomy & 31 \\
\hline 2 & $\mathrm{~F}$ & 69 & Right & 3.1 & 1.1 & $35 \%$ & $x$ & 12 & $x$ & \\
\hline 3 & $\mathrm{~F}$ & 79 & Left & 7.1 & 2.5 & $35 \%$ & $x$ & 12 & $x$ & \\
\hline 4 & $M$ & 64 & Right & 0.8 & 0.3 & $37 \%$ & $x$ & 13.5 & $x$ & \\
\hline 5 & $\mathrm{~F}$ & 63 & Right & 14.3 & 7.3 & $51 \%$ & $x$ & 11 & Craniotomy & 37 \\
\hline
\end{tabular}

VPS: ventriculoperitoneal shunt; Mx: management. 

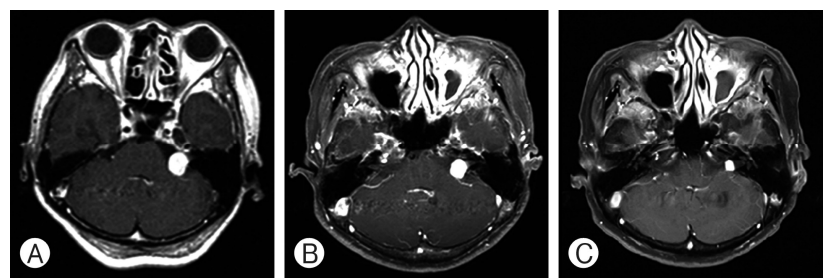

Fig. 1. A series of magnetic resonanceimages, axial $\mathrm{T} 1$-weighted images with contrast, showing cystic mass,obtained in a 58-yearold female with tinnitus and vertigo at left cerebellopontine angle vestibular schwannoma. (A) Before the Gamma knife radiosurgery (GKRS), tumor volume was $2.5 \mathrm{~cm}^{3}$. (B) After 43 months, the tumor volume was decreased. (C) The tumor was more decreased after 94 months from GKRS.
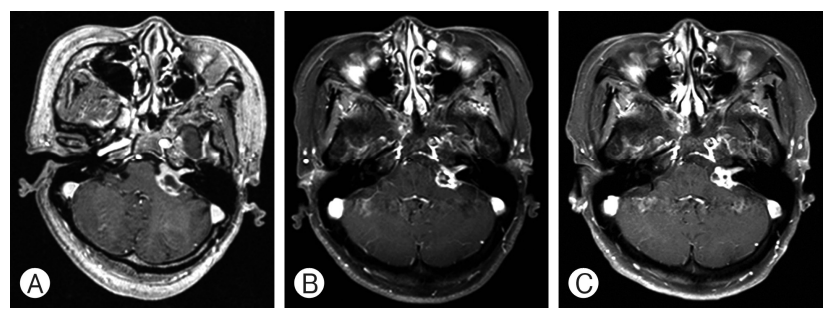

Fig. 2. A series of magnetic resonanceimages, axial $\mathrm{Tl}$-weighted images with contrast, showing cystic mass, obtained in a 63-yearold female with hearing disturbance at right cerebellopontine angle vestibular schwannoma. (A) Before the Gamma knife radiosurgery (GKRS), tumor volume was $5.2 \mathrm{~cm}^{3}$ and cyst volume was $1.6 \mathrm{~cm}^{3}$. (B) After 14 months, the tumor and cyst volume was decreased. (C) The tumor was stable until 52 months from GKRS.
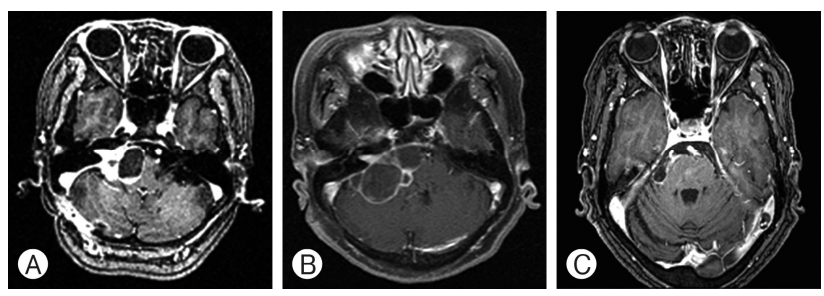

Fig. 3. A series of magnetic resonance images, axial $\mathrm{Tl}$-weighted images with contrast, showing cystic mass enlargement after radiosurgery, obtained in a 63-year-old woman with right cerebellopontine angle vestibular schwannoma. (A) Before the Gamma knife radiosurgery, tumor volume was $14.3 \mathrm{~cm}^{3}$ and cyst volume was $7.3 \mathrm{~cm}^{3}$. (B) After 29 months, the cyst was further enlarged. (C) Craniotomy for tumor and cyst removal due to aggravated dizziness and facial nerve palsy.

is higher tumor and cyst volume. Examples of MR scans for each groups are illustrated in Figs. 1, 2 and 3, respectively.

\section{DISCUSSION}

CVS may represent a unique subtype of tumor. Generally
CVSs are believed by some to have a more aggressive course with more rapid neurological deterioration and less predictable tumor than SVSs ${ }^{12)}$. The pathological mechanism of cyst development in VS is unclear thus far. Isolated or repeated intratumoral hemorrhage, hyaline, fatty, and mucinous degeneration and microcystic change have all been proposed as possible mechanisms of cyst formation. Besides rapid progression, CVS was usually associated with an increased risk of operative damage to the facial nerve, surgery-related complications, and mortality compared with SVS ${ }^{11}$.

The biological mechanisms underlying the progression of CVS after GKRS are poorly understood. Possible mechanisms of CVS expansion after GKRS include osmotic mechanisms from vascular damage, which can induce extravasation of serum proteins into the extracellular matrix. Transient radiation-induced elevation in protein levels may also increase transudation from tumor vessels. Together, these factors increase the osmotic force favoring fluid accumulation in cystic spaces $^{8)}$. Charabiet al. ${ }^{3)}$, mentioned that the formation and progression of cysts were due to the degeneration of tumor tissue. Ki-67 immunostaining of CVS revealed that rapid volume increase was more likely to due to an increase in the cyst volume than to a high growth rate of tumor cells ${ }^{3)}$.

GKRS has become more popular during the last decade, and promising clinical results have been reported for treatment of small- to median sized VSs ${ }^{10)}$. Published results of GKRS for the treatment of VS, treatment failure rate were $1.4 \%^{4)}$ (defined as need for microscopic surgery) and 10\% ${ }^{6}$ (defined as need for tumor enlargement). Compared with SVSs, CVSs tend to behave more unpredictably after GKRS, necessitating surgical removal. A limited number of studies on radiosurgery as the primary treatment for large or CVSs have been conducted, and microsurgery is generally considered the first choice ${ }^{2,5)}$. Delsanti and Régis) ${ }^{5)}$ reported 54 CVSs treated with GKRS, and the failure rate (defined as need for a second procedure), was $6.4 \%$, approximately 3 times what they reported for SVSs. In our study, the tumor progression rate of group 3 was $5 \%$ and group 1 was $18.5 \%$. In group $3,0.6 \%$ of patients needed salvage microscopic surgery because of tumor progression and $7.4 \%$ of patients in group 1 , and $11.1 \%$ of group 2 . This is similar to other studies' results, and CVS shows higher treatment failure when treated with GKRS. For large or symptoms of mass effect and hydrocephalus are presents with VSs, microsurgery is the first option ${ }^{11)}$. And it has been recently suggested that for asymptomatic patient, multisession stereotactic radiosurgery, compared with single-fraction radiosurgery, results in better hearing preservation and, in the case of larger VSs, fewer side effects due to better sparing of the normal tissues and organs at risk ${ }^{22}$. 


\section{CONCLUSION}

In this study we found that GKRS for large CVS was associated with poor treatment outcome. Further study is needed to identify the mechanism of the tumor progression of CVS after GKRS.

\section{REFERENCES}

1. Benech F, Perez R, Fontanella MM, Morra B, Albera R, Ducati A: Cystic versus solid vestibular schwannomas: a series of 80 grade III-IV patients. Neurosurg Rev 28:209-213, 2005

2. Casentini L, Fornezza U, Perini Z, Perissinotto E, Colombo F: Multisession stereotactic radiosurgery for large vestibular schwannomas. J Neurosurg 122:818-824, 2015

3. Charabi S, Klinken L, Tos M, Thomsen J: Histopathology and growth pattern of cystic acoustic neuromas. Laryngoscope 104: 1348-1352, 1994

4. Chopra R, Kondziolka D, Niranjan A, Lunsford LD, Flickinger JC: Long-term follow-up of acoustic schwannoma radiosurgery with marginal tumor doses of 12 to $13 \mathrm{~Gy}$. Int J Radiat Oncol Biol Phys 68:845-851, 2007
5. Delsanti C, Régis J: Cystic vestibular schwannomas. Neurochirurgie 50:401-406, 2004

6. Friedman WA: Linear accelerator radiosurgery for vestibular schwannomas. Prog Neurol Surg 21:228-237, 2008

7. Gal TJ, Shinn J, Huang B: Current epidemiology and management trends in acoustic neuroma. Otolaryngol Head Neck Surg 142:677-681, 2010

8. Kameyama S, Tanaka R, Kawaguchi T, Fukuda M, Oyanagi K: Cystic acoustic neurinomas: studies of 14 cases. Acta Neurochir (Wien) 138:695-699, 1996

9. Kanzaki J, Tos M, Sanna M, Moffat DA, Monsell EM, Berliner $\mathrm{KI}$ : New and modified reporting systems from the consensus meeting on systems for reporting results in vestibular schwannoma. Otol Neurotol 24:642-648, 2003

10. Kondziolka D, Lunsford LD, McLaughlin MR, Flickinger JC: Long-term outcomes after radiosurgery for acoustic neuromas. N Engl J Med 339:1426-1433, 1998

11. Samii M, Gerganov VM, Samii A: Functional outcome after complete surgical removal of giant vestibular schwannomas. J Neurosurg 112:860-867, 2010

12. Thakur JD, Khan IS, Shorter CD, Sonig A, Gardner GL, Guthikonda B, et al.: Do cystic vestibular schwannomas have worse surgical outcomes? Systematic analysis of the literature. Neurosurg Focus 33:E12, 2012

13. Whitmore RG, Urban C, Church E, Ruckenstein M, Stein SC, Lee JY: Decision analysis of treatment options for vestibular schwannoma. J Neurosurg 114:400-413, 2011 